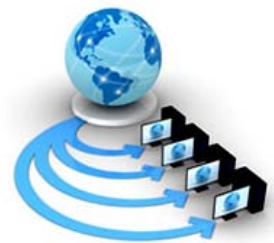

Volume 8, No. 7, July - August 2017

International Journal of Advanced Research in Computer Science

REVIEW ARTICLE

Available Online at www.ijarcs.info

\title{
A REVIEW ON VARIOUS ROUTING PROTOCOLS BASED ON CLUSTERING IN WSN
}

\author{
Er. Simranpreet Kaur \\ M.Tech Scholar Department of Computer Science \& \\ Engineering, Amritsar College of engineering and \\ technology, Amritsar, India
}

\author{
Er. Shivani Sharma \\ Associate Professor, Department of Computer Science \& \\ Engineering, Amritsar College of engineering and \\ technology, Amritsar, India
}

\begin{abstract}
Wireless sensor network (WSNs) are promising while important as well as common strategies to furnishing pervasive computing surroundings for several applications. Unbalanced use of power is surely a normal problem in WSNs, described as multi-hop routing as well as many-to-one visitors pattern. This irregular power dissipation might significantly minimize the system lifetime. So in the effort to enhance the network life-time along with minimizing energy dissipation various energy-efficient routing protocols are designed for the actual sensor networks. Hierarchical routing follows the clustering mechanism which is considered to be efficient in terms of energy and scalability. In this paper, our mainly focused is on the energy efficient hierarchical cluster-based routing protocols. The main objective of this paper is to represent the comparison of clustering hierarchical routing protocols that periodically selects the cluster head according to a hybrid of their residual energy.
\end{abstract}

Keywords: Wireless sensor network (WSN); Clustering; Routing protocols; LEACH and NEAHC.

\section{INTRODUCTION}

Wireless Sensor Networks (WSN) can be approach of hundreds or even several minimal level indicator centers which have limitations of recognizing, working right up far off communications between each various other and also doing computational and also be prepared procedures [1]. Each and every hub position can be of just one or simply a several sensor. Most of these indicator hub issues will be minimal within determine and also bring over the air correspondence. Sensor / probe frameworks get a broad set up of employments and also systems having incomprehensibly fluctuating personal needs and also qualities. The particular indicator systems can be utilized began this morning an unfathomable selection of career fields like army atmosphere, disaster administration, living space observing, medicinal and also cultural insurance policies, mechanical career fields, house systems, specific potion, healthy, radiological, nuclear, and also shaky substance and so on [1]. Structure and also topology of WSN may vary from straightforward star technique to a advanced multi-hop instant fine mesh network. Strength wants, constrained tools, decreased dependability, and a usually increased fullness and also range of disappointment modems will be couple of the issues to be considered when creating events in order to use within indicator systems [2].

This sensing unit centre details might consult amongst their selves applying radio signs. An online sensing unit centre will be set up by using acknowledging plus control units, radio handsets plus drive segments. The person centre details with a remote sensing unit platform (WSN) will be fundamentally reference limited: they've already required controlling velocity, stockpiling almost plus correspondence information and facts transmission. Following the sensing unit focuses will be presented, they can be to blame for self- organizing the proper platform place most of the time by using multi-bounce correspondence by using them. When this occurs in your community available alarms get started meeting information of interest. Out of the way sensing unit cool gadgets similarly interact to concerns delivered at a "control web page" to complete particular guidelines or even allow detecting tests. Figure 1 displays any ordinary simple distant sensing unit system. When can be seen, a total distant sensing unit technique typically makes up of a number of platform programs, various sensing unit sites, plus the stop client.

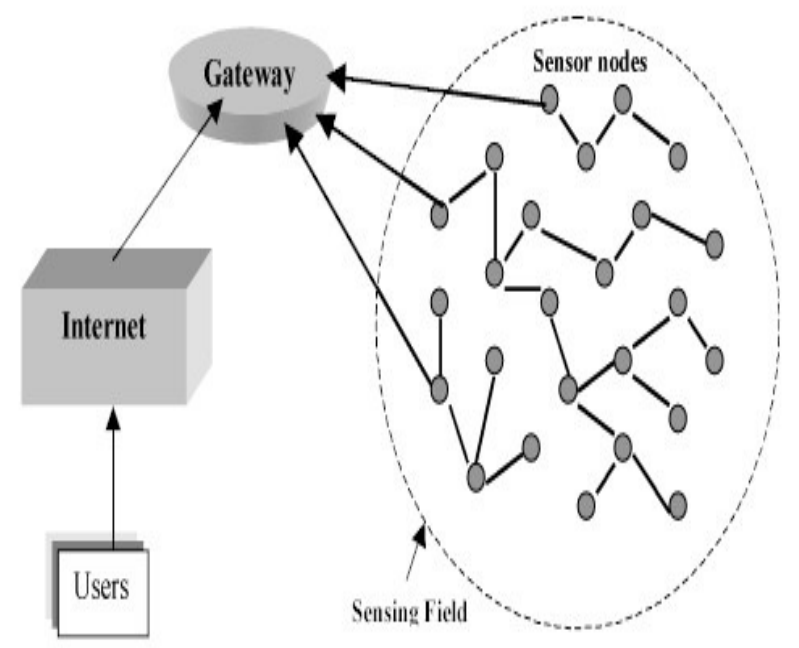

Figure 1: A Simple Wireless Sensor Networks [2]

\section{A. CLUSTERING}

Clustering technique is amongst the well-liked components where nodes decide on a Cluster Mind $(\mathrm{CH})$ regarding communication. Just about all nodes distributed his or her 
details to $\mathrm{CH}$, whereby, that group details and also deliver on the Platform Train station (BS). Solely few nodes are employed distributed most importantly distance thus, a lesser amount of energy will be consumed. The key thought of clustering will be to reduce this multi-level traffic from node to starting station. Nodes include constrained life of the battery thus to shield energy clustering procedure appeared to be released; where from thousands of nodes few nodes become chaos brain and so they manage the whole network. Cluster brain is often a node which is reliable regarding manage chaos, acquire details from nodes while in the chaos and also contact sink. By utilizing clustering technique it has been viewed that there's massive volume of your energy that is saved. Clustering can be done with 2 types of sites: it may be involving two kinds that may be homogeneous and also heterogeneous networks. Within homogeneous sites, preliminary energy of the nodes will be exact when in heterogeneous multi-level; all of nodes include distinct preliminary energy.

\section{ENERGY EFFICIENT ROUTING PROTOCOLS IN WSN}

\section{A. Hybrid Energy-Efficient Distributed clustering (HEED)}

HEED is actually a multi-hop WSN clustering criteria of which virtually delivers your energy-efficient clustering routing by utilizing occasionally specifically anxiousness of energy. Entirely completely different from LEACH around the procedures by using $\mathrm{CH}$ devotion, HEED will not go with nodes seeing that $\mathrm{CHs}$ randomly. The best way of chaos construct is done dependant on your a mix of both collection by using a number of parameters. On the list of factors relies upon round the node's still left in excess of vitality, as well as the other parameter could possibly be the intra-cluster interacting expenditure. Inside of HEED, favored CHs have very good normal still left vitality compared to MNs. In addition, amongst the chief wanted objectives by using HEED will be to collect sometimes present $\mathrm{CHs}$ everywhere in the systems [9]. In addition, no matter what sensation this a couple of nodes, in just each other's interacting assortment, turn into CHs along, nonetheless the potential for of which sensation can be quite small within HEED. Inside of HEED, CHs have normal times favored dependant on a number of critical factors: continuing vitality plus intra-cluster interacting price tag inside the prospect nodes .

\section{B. Low Energy Adaptive Clustering Hierarchy (LEACH)}

LEACH is a well known cluster protocol which elect $\mathrm{CH}$ on random basis to communicate data with sink, it uses TDMA to transmit data between sink and CH's.. Around LEACH the nodes sort nearby clusters and among the nodes performing being a nearby drain or even group head. In case exactly the same node would certainly stay because group go all over the working of your system, it'd kick the bucket easily because of the in depth download from the contributing sensors from the group [2]. In every iteration $\mathrm{CH}$ 's are changed in such a way that one can divide load among nodes in an efficient way. Even more electrical power dissipation is often decreased by means of aggregating the results from many alarm nodes within the group head.

\section{Distributed Weight-based Energy-efficient Hierarchical Clustering protocol (DWEHC)}

DWEHC might be a allocated clustering algorithm formula for instance HEED. The key cause for DWEHC can be to improve HEED as they make nicely balanced chaos sizes as well as improve the intra-cluster topology utilizing posture knowing the nodes. Either just as DWEHC as well as HEED mention a variety of characteristics in addition to no presumptions with regards to multilevel sizes as well as awareness, and using within concern remaining electricity within the approach with regards to $\mathrm{CH}$ selection. Just about all nodes tools DWEHC independently combined with the algorithm formula finishes following numerous iterations which might be utilized for the dispersed manner. Not the same as LEACH as well as HEED, DWEHC creates the multi-level business enterprise meant for intra-cluster communicating as well as limits a mother or father node's amount of children.

\section{Power Efficient Gathering in Sensor Information System (PEGASIS)}

The key plan in PEGASIS will be for every node to obtain coming from in addition to send out to seal others who live nearby in addition to take turns currently being the best choice pertaining to transmitting to your BS. This method will probably distribute the energy fill smoothly on the list of sensing unit nodes from the multi-level [3]. Most of us initially put the nodes at random from the participate in industry, and for that reason, the ith node is at your unique location. A node will probably be sorted produce a string, which can either be done because of the sensing unit nodes by themselves with a greedy algorithm formula beginning a number of node. However, the BS can easily estimate this kind of string in addition to transmit that to everyone the sensing unit nodes [3]

\section{E. Multipath routing}

Single direction course-plotting standards in sensor network are created to discover a single direction from your source/destination pair. With the contrary, multipath courseplotting consists of getting many walkways in between the fundamental cause and location nodes. These many walkways can often resolve many trade-offs in these communities and have capacity for using the powerful design associated with WSNs. Within this, we all talk about some gains and benefits of multipath routing. At the same time, we all talk about many conditions crop up if you use multipath course-plotting in wireless sensor networks.

\section{RELATED WORK}

Wang Ke et.al (2016)[1]proposed mobile sensing unit systems (WSNs) are promising when crucial and also wellknown means of offering pervasive computing surroundings for several applications. Unbalanced power consumption is usually an purely natural injury in WSNs, seen multi-hop redirecting and a many-to-one targeted traffic pattern. That uneven power dissipation could tremendously lower community lifetime. During this newspaper, most of us propose some sort of fresh power aware hierarchical clusterbased (NEAHC) redirecting method with 2 goals: reducing the whole power consumption and also making certain fairness of one's consumption amongst nodes. Izadi, Davood 
et.al (2013) [6] studied instant indicator Cost Per Action(CPA) affiliate networks power conservation within the CPA affiliate networks is still one of the most crucial study challenges. Just one strategy popular to extend the particular multi-level lifelong is through aggregating details in the bunch mind (CHs). Nevertheless, there is certainly possibility the CHs may be unsuccessful and performance incorrectly because of number of explanations just like energy instability. Throughout the disaster, the particular CHs cannot acquire and transfer details correctly. This particular has effects on the particular overall performance on the WSN. Within this newspaper they recommended the self-configurable clustering system to discover the particular disordered $\mathrm{CHs}$ and substitute them along with other nodes. Leu, Jenq-Shiou et.al (2015) [2] surveyed a brand new localized power mindful clustering process working with remote nodes with regard to WSNs, known as Local Electrical power Knowledgeable Clustering along with Out of the way Nodes (REAC-IN). With REAC-IN, CHs are generally decided on based upon weight. Fat is based on the extra power of every alarm and the localized regular power of devices in just about every cluster. Badly developed sent out clustering algorithms could cause nodes for being remote via CHs. Like remote nodes connect using the destroy by taking excess amount of energy. Khediri et.al (2014)[3] consisting of multitude of low-cost as well as minor homogenous alerts nodes hooked up by an invisible circle which assemble files being dealt with in your neighborhood as well as relayed for the torpedo node by multi-hop wi-fi transmission. Also, such troubles are certainly essential because of machines methods demands such as powerful power, stock options restriction as well as use of network. Quite a few solutions ended up offered to attenuate the website traffic within network. Clustering algorithms have already been trusted to relieve power consumption. Thakkar et.al (2014) [4] proposed the planning of any multi-hop Wireless Sensor / probe System (WSN) will depend on the requirements the root realizing application. The leading aim with WSNs will be to watch real phenomenon of interest inside of a presented place of interest utilizing devices and provide accumulated details in order to sink. This WSN consists of a huge number of strength, communicating, and also computational limit nodes, to get rid of strength constrains, and also exchanging or recharging the electric batteries with the WSN nodes can be an impossible process, after they are stationed inside of a violent environment. Consequently, in order to keep the community alive providing possible, communicating between your WSN nodes has to be done with download balancing. Wagh et.al (2014)[5] proposed the actual wifi sensor / probe systems are built to mount the actual clever circle software or even circle pertaining to unexpected emergency solutions, wherever our relationship is not possible. The nodes around wifi sensor / probe systems have to home coordinate according to you needs by overseeing environments. Because sensor / probe nodes usually are used in the inaccessible spot for special mission, it is sometimes complicated to exchange or even revitalize the actual nodes battery. Hence giving her very troubles to develop the actual sensor / probe circle for max time period time period of circle as well as low power function with the nodes. Izadi et.al (2013)[6] reviewed important developments with instant warning systems (WSNs), power conservation is always one of the most significant investigation challenges. Correct group with nodes (clustering) is probably the big approaches to expand the actual life expectancy with the whole multilevel as a result of aggregating data within the chaos head. This chaos scalp will be the anchor with the entire cluster. Actually when a chaos scalp ceases to attain it is function, the actual acquired and amassed data by means of chaos scalp could be lost. Additionally, the force consumption subsequent strong devices out of solutions to platform stops will likely be increased. Izadi, Davood et.al (2012) [7] analyzed a mobile warning community (WSN) is usually several alarms which might be geographically sent out as well as connected through mobile networks. Devices collect more knowledge about the condition of natural world. In that case, following running forward them to the leading destination. In order to supply this specific service, there are several issues with transmission techniques that need to be explored. Encouraging service quality (QOS) will probably represent significant relevance for persistent WSNs this be your community structure connected with diversified applications. Mhemed et.al (2012) [8] studied the significant progress with cellular warning cpa networks (WSNs),energy resource efficiency stays probably the most significant investigation challenges. Researchers have investigated architectures and also topologies which allow cost effective functioning regarding WSNs. One of the popular methods labels on homeopathic products is clustering. While researchers have investigated cluster scalp variety. Lee et.al (2012) [9] surveyed this clustering presents a good way to extend this time of WSNs. Latest clustering ways typically work with a couple of approaches: picking chaos leads with increased recurring vitality, and twisting chaos leads routinely, for you to distribute the action use among nodes throughout just about every chaos and increase this system lifetime. Even so, a lot of the preceding algorithms never have considered this envisioned recurring vitality, the predicated outstanding vitality as selected like a chaos mind and working around. Some sort of fuzzy-logic-based clustering approach with an extension cord towards the vitality predication may be planned to extend this time of WSNs simply by smoothly disbursing this workload. Stephanie Lindsey et.al [10] proposed "Power-effective social occasion in sensor data frameworks" .It is almost ideal convention in addition to is desirable over LEACH convention. Inside the proposed technique, each and every revealing unit hub sends that features nearby neighbor solely as well as to sends information to the BS around each turns, thus small way of measuring energy is obviously placed in each and every round. PEGASIS is dependent on various sequence of calculation which is turn an ideal for information gathering throughout detecting unit systems. 


\section{COMPARISON TABLE}

TABLE 1

Comparison of Various Techniques

\begin{tabular}{|c|c|c|c|c|}
\hline Protocol & Mobility & Hop Count & $\begin{array}{c}\text { Energy } \\
\text { Efficiency }\end{array}$ & Scalability \\
\hline Leach & Fixed & Single hop & Low & Good \\
\hline Leach- C & Fixed & Single hop & Very High & Good \\
\hline Leach-F & Fixed & Single hop & High & Limited \\
\hline EARP & Stationary & Multi hop & High & High \\
\hline SA-Leach & Variable & Single hop & High & Good \\
\hline O-Leach & Variable & Single hop & High & Good \\
\hline EEZECR & Variable & Multi hop & High & Good \\
\hline ELCH & Fixed & Single hop & High & Limited \\
\hline EEHC & Fixed & Single Hop & Low & Very Low \\
\hline O-Leach & Variable & Single hop & Very High & Very good \\
\hline Cog-Leach & Variable & Single hop & Very High & Good \\
\hline Leach-SM & Variable & Single hop & Very High & Good \\
\hline AQM-Leach & Variable & Single hop & High & Limited \\
\hline I-Leach & Variable & Multi hop & Very High & Good \\
\hline E-Leach & Variable & Single hop & Moderate & Good \\
\hline Multi-level Leach & Variable & Multi hop & High & Medium \\
\hline Op-Leach & Variable & Single hop & Moderate & Medium \\
\hline Leach with Mobile Sink & Variable & Multi hop & High & Medium \\
\hline PR-Leach & Variable & Single hop & Moderate & Good \\
\hline Q-Leach & Variable & Multi hop & Very High & Good \\
\hline A-Leach & Fixed & Single hop & Very High & Good \\
\hline Leach-A & Fixed & Single hop & Very High & Good \\
\hline Leach-B & Fixed & Single hop & Very High & Good \\
\hline Leach-C & Fixed & Single hop & Very High & Very Good \\
\hline Leach-E & Variable & Single hop & Very High & Very Good \\
\hline Leach-F & Variable & Single hop & Very High & Limited \\
\hline Leach-M & Mobile BS and Nodes & Multi hop & Very High & Very Good \\
\hline
\end{tabular}




\begin{tabular}{|c|c|c|c|}
\hline Leach-L & Fixed & Multi hop & Very High \\
\hline Leach-S & Fixed & Single hop & Very High \\
\hline V-Leach & Fixed & Single hop & Very High \\
\hline TL-Leach & Fixed & Single hop & Very High Good \\
\hline Leach-E & Fixed & Single hop & High Gery Good \\
\hline
\end{tabular}

In this comparison table 1 ,we compare the various routing protocols based on cluster based routing but in all routing protocols the other parameters like mobility, hop count, energy efficiency and scalability varies.

\section{CONCLUSION}

Wireless network composed of spatially spread autonomous system working with detectors to evaluate physical as well as the environmental conditions. The WSN system contains a path which offers wireless connection back to the community along with distributed nodes. This specific paper shows the comparison of various techniques of routing protocols in Wireless Networks. This review has reveals that the majority of the of established methods neglected the the effects of the mobile sink in energy efficient routing protocols, the issue regarding lossless data compression continues to be neglected from the majority of analysts and no optimization method is regarded with the effective path selection in NEAHC. Therefore in near future we will purpose technique based on energy efficient protocols to overcome the restrictions of the existing energy efficient protocols by using the compressive sensing as well as evolutionary optimization based tree structured. Various analytics is going to be utilized to measure the enhancement throughout proposed technique over the existing energyefficient protocols

\section{REFERENCES}

[1] Ke, Wang, et al. "Energy aware hierarchical cluster-based routing protocol for WSNs." The Journal of China Universities of Posts and Telecommunications 23.4 (2016): 46-52.Izadi, Davood, JemalAbawajy, and Sara Ghanavati. "An Alternative Clustering Scheme in WSN." (2015).

[2] Leu, Jenq-Shiou, Tung-Hung Chiang, Min-Chieh Yu, and Kuan-Wu Su. "Energy Efficient Clustering Scheme for Prolonging the Lifetime of Wireless Sensor Network With
Isolated Nodes." Communications Letters, IEEE 19, no. 2 (2015): 259-262.

[3] Khediri, Salim EL, NejahNasri, Anne Wei, and AbdennaceurKachouri. "A new approach for clustering in wireless sensors networks based on LEACH."Procedia Computer Science 32 (2014): 1180-1185.

[4] Thakkar, Ankit, and KetanKotecha. "Cluster head election for energy and delay constraint applications of wireless sensor network." Sensors Journal, IEEE 14, no. 8 (2014): 2658-2664.

[5] Wagh, Sushama, and Ranga Prasad. "Maximizing lifetime of wireless sensor networks using genetic approach." In Advance Computing Conference (IACC), 2014 IEEE International, pp. 215-219. IEEE, 2014.

[6] Izadi, Davood, JemalAbawajy, and Sara Ghanavati. "A new energy efficient cluster-head and backup selection scheme in WSN." In Information Reuse and Integration (IRI), 2013 IEEE 14th International Conference on, pp. 408-415. IEEE, 2013.

[7] Izadi, Davood, JemalAbawajy, and Sara Ghanavati. "Quality control of sensor network data." In Advances in Automation and Robotics, Vol. 1, pp. 467-480. Springer Berlin Heidelberg, 2012.

[8] Mhemed, Rogaia, NaumanAslam, William Phillips, and Frank Comeau. "An energy efficient fuzzy logic cluster formation protocol in wireless sensor networks." Procedia Computer Science 10 (2012): 255-262.

[9] Lee, Jin-Shyan, and Wei-Liang Cheng. "Fuzzy-logic-based clustering approach for wireless sensor networks using energy predication." Sensors Journal, IEEE12, no. 9 (2012): 28912897.

[10] Lindsey, Stephanie, and Cauligi S. Raghavendra. "PEGASIS: Power-efficient gathering in sensor information systems." Aerospace conference proceedings, 2002. IEEE. Vol. 3. IEEE, 2002. 